\title{
Abscissas and Weight Coefficients for Lobatto Quadrature
}

\author{
By H. H. Michels
}

1. Introduction. Recently, the numerical evaluation of certain collision integrals was studied using several different mechanical quadrature formulas, including Gaussian quadrature of high order [1,2] and various Newton-Cotes formulas. It was found that high accuracy could not easily be obtained, owing to the particular behavior of the integrand at the end points of integration, and it seemed likely that a "closed" type Gaussian formula of high order might be more efficient for this particular application.

The existence of Gaussian-type quadrature formulas with one or more prescribed abscissas has been investigated by Lobatto [3] and Radau [4]. For the case where both ends of the integration interval are preassigned (Lobatto quadrature), the free abscissas and the corresponding weight coefficients have been evaluated by Radau [5] up to order 11. More recently abscissas and weights for Lobatto quadrature have been reported by Rabinowitz [6] for selected odd order up to 65. In some cases, however, an even-order quadrature formula may be desired and the results for such formulas of high order are reported in this communication.

2. Method of Computation. We are concerned with the Lobatto quadrature formulas of order $n$ normalized by a change of variables to the interval $(-1,1)$

$$
\int_{-1}^{+1} f(x) d x=H_{1} f(-1)+\sum_{k=2}^{n-1} H_{k} f\left(x_{k}\right)+H_{n} f(+1) .
$$

Formula (1) is exact for all polynomials $f(x)$ of degree $\leqq 2 n-3$, whereas Gaussian quadrature rules are exact for degree $\leqq 2 n-1$. However, if the function $f(x)$ is zero at both ends of the integration interval, only $r-2$ ordinates are involved in the calculation and a higher effective degree of precision is obtainable than if an open Gaussian type formula is used. The free abscissas $x_{k}(k=2,3, \cdots, n-1)$ are the zeros of the first derivative of the Legendre polynomial of order $n-1$

$$
P_{n-1}^{\prime}\left(x_{k}\right)=0 .
$$

The corresponding weight coefficients $H_{k}$ can be found from the expression

$$
H_{k}=\frac{2}{n(n-1)\left[P_{n-1}\left(x_{k}\right)\right]^{2}}
$$

where $P_{n-1}\left(x_{k}\right)$ is the normalized Legendre polynomial of order $n-1$. The weights corresponding to the fixed abscissas at $x= \pm 1$ are found to be

$$
H_{-1}=H_{+1}=\frac{2}{n(n-1)} .
$$

Received September 19, 1962 
A first approximation to the zeros of the derivative of the Legendre polynomial $P_{n}^{\prime}(x)$ can be obtained in several ways. It can be shown [7] that

$$
\lim _{n \rightarrow \infty}\left\{n^{-m} P_{n}{ }^{m}\left[\cos \left(\frac{x}{n}\right)\right]\right\}=J_{m}(x) .
$$

Since the zeros of $P_{n}{ }^{\prime}(x)$ are the same as those of the associated Legendre polynomial $P_{n}{ }^{1}(x)$ through the relation

$$
P_{n}{ }^{1}(x)=\left(x^{2}-1\right)^{1 / 2} P_{n}^{\prime}(x)
$$

equation (5) can be used to relate the zeros of $P_{n}{ }^{1}(x)$ to the successive zeros of the Bessel function $J_{1}(x)$.

A better approximation to the zeros of $P_{n}^{\prime}(x)$ can be obtained by making use of the inequalities derived by Szegö [8] for the zeros of the generalized Jacobi polynomial $P_{n}{ }^{(\alpha, \beta)}(x)$. An examination of the upper and lower bounds of the zeros of $P_{n-1}^{\prime}(x)$ showed that two or three decimal places could be established using the relation

$$
x_{n, k}=\cos \left\{\frac{j_{1, k}}{\left[(n-1 / 2)^{2}+\left(\frac{\pi^{2}-4}{4 \pi^{2}}\right)\right]^{1 / 2}}\right\}
$$

where $j_{1, k}$ are the successive zeros of the Bessel function $J_{1}(x)$. These initial approximations to the roots were improved using Newton-Raphson iteration

$$
\left(x_{n, k}\right)_{i+1}=\left(x_{n, k}\right)_{i}-\frac{P_{n-1}^{\prime}\left(x_{n, k}\right)_{i}}{P_{n-1}^{\prime \prime}\left(x_{n, k}\right)_{i}} .
$$

The Legendre polynomials and their derivatives were computed using the recursion formulas

$$
\begin{aligned}
P_{n+1}(x)= & \left(\frac{2 n+1}{n+1}\right) x P_{n}(x)-\left(\frac{n}{n+1}\right) P_{n-1}(x) \\
P_{0}(x)=1 ; \quad P_{1}(x)=x & \left(\frac{2 n+1}{n}\right) x P_{n}^{\prime}(x)-\left(\frac{n+1}{n}\right) P_{n-1}^{\prime}(x) \\
P_{n+1}^{\prime}(x)= & P_{0}^{\prime}(x)=0 ; \quad P_{1}^{\prime}(x)=1 \\
P_{n+1}^{\prime \prime}(x)= & \left(\frac{2 n+1}{n-1}\right) x P_{n}^{\prime \prime}(x)-\left(\frac{n+2}{n-1}\right) P_{n-1}^{\prime \prime}(x) \\
& P_{1}^{\prime \prime}(x)=0 ; \quad P_{2}^{\prime \prime}(x)=3 .
\end{aligned}
$$

The weight coefficients were computed directly using equations (3) and (4).

3. Results. Abscissas and weights for Lobatto quadrature are presented in Table I for order $n=3(1) 16,24,32,40,48,64,80,96$. All computations were performed on an IBM 7090 digital computer using extended precision routines. The tolerance for iteration on the roots was set at $1 \times 10^{-22}$. Several hand calculations of the roots and weight coefficients were performed. Complete agreement 
TABLE I

Abscissas and Weight Coefficients for Lobatto Quadrature

Abscissas

Weights

$n=3$

\section{$1.0000000000 \quad 0000000000$ \\ $0.0000000000 \quad 0000000000$ \\ $1.0000000000 \quad 0000000000$ \\ $0.4472135954 \quad 9995793928$ \\ $1.0000000000 \quad 0000000000$ \\ $0.6546536707 \quad 0797714380$ \\ $0.0000000000 \quad 0000000000$}

1.0000000000

0.7650553239

0.2852315164

1.0000000000

0.8302238962

0.4688487934

0.0000000000

1.0000000000

0.8717401485

0.5917001814

0.2092992179

1.0000000000

0.8997579954

0.6771862795

0.3631174638

0.0000000000

1.0000000000

0.9195339081

0.7387738651

0.4779249498

0.1652789576

1.0000000000

0.9340014304

0.7844834736

0.5652353269

0.2957581355

0.0000000000

1.0000000000

0.9448992722

0.8192793216

0.6328761530

0.3995309409

0.1365529328

1.0000000000

0.9533098466
0000000000

2946469285

8064509631

0000000000

7856692987

7071421380

0000000000

0000000000

0960661534

3314230214

0247886877

0000000000

1146015731

1073775345

2617815871

0000000000

0000000000

6645881383

0550507500

1044449566

6638702463

0000000000

0805913433

6314441862

9620500647

8693939143

0000000000

0000000000

2288222341

4400667835

3186067766

6534893226

549275.5486

0000000000

4216391190
0.3333333333

1. 3333333333

$n=4$

$0.1666666666 \quad 6666666667$

$0.8333333333 \quad 3333333333$

$n=5$

$0.1000000000 \quad 0000000000$

$0.5444444444 \quad 4444444444$

$0.7111111111 \quad 1111111111$

$n=6$

$0.0666666666 \quad 6666666667$

$0.3784749562 \quad 9784698032$

$0.5548583770 \quad 3548635302$

$n=7$

0.0476190476

0.2768260473

0.4317453812

0.4876190476

$n=8$

0.0357142857

0.2107042271

0.3411226924

0.4124587946

$n=9$

0.0277777777

0.1654953615

0.2745387125

0.3464285109

0.3715192743

$n=10$

0.0222222222

0.1333059908

0.2248893420

0.2920426836

0.3275397611

$n=11$

0.0181818181

0.1096122732

0.1871698817

0.2480481042

0.2868791247

0.3002175954

$n=12$

0.0151515151

0.0916845174

0.1579747055

0.2125084177

0.2512756031

0.2714052409

$n=13$

$0.0128205128 \quad 2051282051$

$\begin{array}{lll}0.0778016867 & 4681892779\end{array}$

1904761905

6156594801

0986262342

1904761905

1428571429

4350603938

8350436476

5870388157

7777777778

6080552505

0016173528

7304634512

7641723356

2222222222

5107011113

6312645212

7968375788

8389745666

8181818182

6699486446

8030520411

6402831404

7900808868

5569069379

5151515152

1319613067

6437011517

6102114536

9920128029

1069617700 
$\begin{array}{ll}0.8463475646 & 5187231687 \\ 0.6861884690 & 8175742607 \\ 0.4829098210 & 9133620175 \\ 0.2492869301 & 0623999257 \\ 0.0000000000 & 0000000000\end{array}$

1.0000000000

0.9599350452

0.8678010538

0.7288685990

0.5506394029

0.3427240133

0.1163318688

1.0000000000

0.9652459265

0.8850820442

0.7635196899

0.6062532054

0.4206380547

0.2153539553

0.0000000000

1.0000000000

0.9695680462

0.8992005330

0.7920082918

0.6523887028

0.4860594218

0.2998304689

0.1013262735

1.0000000000

0.9867305535

0.9557482209

0.9077056751

0.8434640701

0.7641704824

0.6712401052

0.5663313579

0.4513163732

0.3282476133

0.1993212533

0.0668379937

1.0000000000

0.9926089339

0.9752946904

0.9482848384

0.9118499390

0.8663524760

0.8122447317

0.7500644939

0.6804297556

0.6040325871

0.5216322628

0.4340477172
0000000000

3034725100

9132614059

2864705532

4271284504

8370386766

0000000000

0383857280

2297629883

5181520070

6984571112

1367248092

6379423823

0000000000

0000000000

7021793295

9347209299

6181506393

8249308947

8713761178

0076320810

2194944784

0000000000

0516088355

2988635803

1350652200

5487204062

2049330779

6412869984

7929531219

1432261825

7551091203

9083266724

3722857811

0000000000

7276135937

8270922806

1723237808

6373190407

1267551983

7744234455

3667479772

1555081594

4842112614

8156529061

0184693960
6726090135
0.1349819266

0.1836468652

0.2207677935

0.2440157903

0.2519308493

$n=14$

0.0109890109

0.0668372844

0.1165866558

0.1600218517

0.1948261493

0.2191262530

0.2316127944

$n=15$

0.0095238095

0.0580298930

0.1016600703

0.1405116998

0.1727896472

0.1969872359

0.2119735859

0.2170481163

$n=16$

0.0083333333

0.0508503610

0.0893936973

0.1242553821

0.1540269808

0.1774919133

0.1936900238

0.2019583081

$n=24$

0.0036231884

0.0222368534

0.0396316813

0.0563098487

0.0719818620

0.0863690299

0.0992148276

0.1102900868

0.1193971937

0.1263736420

0.1310949418

0.1334768438

$n=32$

0.0020161290

0.0123981065

0.0221995528

0.0317751354

0.0410342015

0.0498852713

0.0582404972

0.0660168772

0.0731371396

0.0795305256

0.0851334979

0.0898903729
8960834912

0355009201

6611008609

0667635646

3344673604

8901098901

9768128463

9871165154

6295214241

7341611864

0977075487

6845705889

2380952381

2860124910

2571806760

0242810946

5360094905

6461335609

2682092013

4881564951

3333333333

0591990540

2593080099

3251409835

0716428081

9170412530

2520358432

7822987149

0579710145

6471120899

3346780947

2464619902

5529398222

6792906822

8408358741

9296860411

0249131903

2802080013

7360394235

6698637760

3225806452

0137384379

8929196462

1091546578

8606272333

3622120701

4805586955

5715454393

0267903264

9210625229

4966823053

5735783307 
Abscissas

Weights

\subsection{5 \\ 0.2468506588 \\ 0.1490985968 \\ 0.0498647250}

1.0000000000

0.9952979292

0.9842662807

0.9670100764

0.9436397649

0.9143033396

0.8791863434

0.8385108227

0.7925339526

0.7415464191

0.6858705850

0.6258584527

0.5618894392

0.4943679781

0.4237209621

0.3503950449

0.2748538167

0.1975748737

0.1190467984

0.0397660708

1.0000000000

0.9967477813

0.9891114700

0.9771488468

0.9609131535

0.9404755493

0.9159254499

0.8873702243

0.8549347448

0.8187608474

0.7790067135

0.7358461791

0.6894679748

0.6400749012

0.5878829421

0.5331203198

0.4760264975

0.4168511320

0.3558529823

0.2932987778

0.2294620522

0.1646219473

0.0990619925

0.0330688647

1.0000000000

0.9981798715

0.9939027267

0.9871926766

0.9780666628

0.9665471103
3888148625

5020530442

1364749491

4659325231

0000000000

4434889690

1750335473

8798852065

4360164257

9020945107

7933983789

7810644074

0155188681

4738441749

8431371383

5525751340

9472264877

2525360665

5555098475

1418087798

1432436652

1891077184

4497109352

0218190015

0000000000

3985746440

1363572789

9083677288

0638159011

3508129520

7624642008

1822009720

3877407027

8560586870

9984382197

1284836876

1109071037

6595713783

4548518103

3277696111

0285707802

3270266991

2890604382

4975926536

7124457033

9809062748

5075011235

6615291109

0000000000

0216321518

0305729237

0274024265

3139607396

6909923352
$0.0937538755 \quad 4681381357$

0.09668560894800260056

$0.0986564365 \quad 4076177717$

$0.0996467715 \quad 0127677764$

$n=40$

$0.0012820512 \quad 8205128205$

$0.0078910115 \quad 8860061376$

$0.0141593075 \quad 4991977315$

$0.0203347590 \quad 6338716185$

$0.0263811906 \quad 5314148626$

$0.0322607179 \quad 2711739549$

$0.0379362437 \quad 0070844699$

$0.0433719081 \quad 9475798101$

$0.0485333538 \quad 4591432514$

$0.0533879519 \quad 7149418296$

$0.0579050119 \quad 8178608340$

$0.0620559764 \quad 7570952497$

$0.0658146022 \quad 2289590266$

$0.0691571262 \quad 7608113446$

$0.0720624163 \quad 0205429522$

$0.0745121042 \quad 3538933858$

$0.0764907024 \quad 3339650625$

$0.0779857016 \quad 0868058073$

$0.0789876499 \quad 2536434419$

$0.0794902127 \quad 6154964087$

$n=48$

$0.0008865248 \quad 2269503546$

$0.0054591926 \quad 0024811922$

$0.0098069319 \quad 7890032805$

$0.0141094906 \quad 0548871324$

$0.0183500364 \quad 7521908147$

$0.0225102637 \quad 3693608450$

$0.0265720325 \quad 9091131712$

$0.0305175976 \quad 2130364253$

$0.0343297117 \quad 0067383698$

0.03799170792672815653

$0.0414875745 \quad 1884729112$

$0.0448020255 \quad 6407161048$

0.04792056815673550066

$0.0508295659 \quad 0594469726$

$0.0535162986 \quad 2867493123$

$0.0559690180 \quad 0514883558$

$0.0581769989 \quad 6927463166$

$0.0601305866 \quad 1684402305$

$0.0618212384 \quad 2997936681$

$0.0632415616 \quad 3503798566$

$0.0643853455 \quad 3160117653$

$0.0652475886 \quad 5172582358$

$0.0658245206 \quad 3100801710$

$0.0661136186 \quad 9600179419$

$n=64$

0.0004960317

0.0030560082

0.0054960162

0.0079212897

0.0103270023

0.0127073991
4603174603

4491249038

0381715690

9004663404

6681532846

9745473520 
TABLE I-Continued

Abscissas

Weights

0.9526622357
0.9364460274
0.9179381735
0.8971839678
0.8742342006
0.8491450345
0.8219778673
0.7927991818
0.7616803834
0.7286976250
0.6939316212
0.6574674503
0.6193943461
0.5798054800
0.5387977327
0.4964714569
0.4529302322
0.4082806112
0.3626318592
0.3160956861
0.2687859740
0.2208184974
0.1723106410
0.1233811116
0.0741496479
0.0247367276

1.0000000000

0.9988386765

0.9961086610

0.9918229201

0.9859884777

0.9786145023

0.9697125241

0.9592964489

0.9473825428

0.9339894093

0.9191379609

0.9028513869

0.8851551171

0.8660767821

0.8456461708

0.8238951831

0.8008577808

0.7765699339

0.7510695652

0.7243964904

0.6965923564

0.6677005761

0.6377662605

0.6068361486

0.5749585341

0.5421831900

0.5085612912

0.4741453343

0.4389890557

0.4031473479

0.3666761732
8866291546

7563416245

1028163083

4585004284

5762749177

4299098500

0751705008

2620813735

0811996779

8883693957

9070484081

1297650421

3843154754

6771842373

1680043899

3605775350

3158118199

8985404453

2626182369

9562580640

1917000399

9695350321

8779297132

5002798616

4611591873

2195872850

0000000000

3092507835

7990519938

8121586819

63353179:2

1691623735

8079561736

56279:8346

4356652296

4788020310

9088049972

7154153396

0238834635

7504981912

5578157538

9510250605

3200733070

7441852493

4070981102

5110723775

6205299908

4097178467

8319665727

7703163943

2701742034

4940380757

5760347898

5899572548

8785790006

0241926342

7705082998
0.0150566839

0.0173691163

0.0196390407

0.0218609035

0.0240292681

0.0261388286

0.0281844226

0.0301610444

0.0320638570

0.0338882038

0.0356296205

0.0372838454

0.0388468305

0.0403147508

0.0416840142

0.0429512698

0.0441134164

0.0451676101

0.0461112710

0.0469420900

0.0476580338

0.0482573503

0.0487385731

0.0491005244

0.0493423184

0.0494633636

$n=80$

0.0003164556

0.0019500843

0.0035089042

0.0050614259

0.0066059324

0.0081400967

0.0096615416

0.0111678973

0.0126568138

0.0141259672

0.0155730640

0.0169958450

0.0183920888

0.0197596154

0.0210962896

0.0224000244

0.0236687842

0.0249005880

0.0260935124

0.0272456949

0.0283553364

0.0294207044

0.0304401355

0.0314120380

0.0323348942

0.0332072634

0.0340277833

0.0347951729

0.0355082340

0.0361658531

0.0367670036
8796144273

8454218159

2324171838

1151806011

4402382671

1433843775

6584851747

9908945068

5772702451

8412539761

2448948628

5980117259

3780773670

8156023712

5080195219

3360181861

4689247092

2594770204

8428905945

2702831645

0222063679

7641454890

2223318512

0750130793

7713957394

2077664644

9620253165

5097755934

0102901840

4958905768

9516823379

2701197166

7369727760

4774327942

2026628430

1119237429

3491764695

4087065425

4689349982

5504496628

7828148968

8605002555

6930063219

2244976823

3860403063

1386453736

5636954355

9572629427

8855621511

1599442732

6912755883

1851378337

6410102158

6204047278

2508140861

9342834538

7314253757 


0.3296324773
0.2920740993
0.2540596823
0.2156485813
0.1769007706
0.1378767504
0.0986374519
0.0592441428
0.0197583310

1.0000000000

0.9991951753

0.9973028330

0.9943310519

0.9902832781

0.9851639321

0.9789785649

0.9717338739

0.9634377002

0.9540990218

0.9437279441

0.9323356897

0.9199345860

0.9065380519

0.8921605834

0.8768177378

0.8605261173

0.8433033505

0.8251680735

0.8061399100

0.7862394497

0.7654882262

0.7439086937

0.7215242027

0.6983589748

0.6744380761

0.6497873908

0.6244335925

0.5984041157

0.5717271260

0.5444314897

0.5165467424

0.4881030574

0.4591312125

0.4296625573

0.3997289787

0.3693628669

0.3385970800

0.3074649086

0.2760000399

0.2442365208

0.2122087217

0.1799512986

0.1474991563

0.1148874102

0.0821513483

0.0493263932

0.0164480637
2342034030 7704882920

8810009988

5741284831

6074369751

0592486577

6914984692

5788193249

4893162978

0000000000

7692604333

1700828646

9061228080

5433043846

2661401114

7778433333

8905729625

8792649710

2054016010

6218866932

9926625583

8504168194

4989011153

0720878924

8235794840

8703298179

5998190621

9491829583

7704236359

5042948018

3952078195

4898009871

6722755296

0000278398

6133855505

5033178220

4307948931

3009891832

3047185738

1484151328

7024456131

4058016068

7730066577

$356545: 3103$

5251961757

4253394460

4986013077

93:08633338

4469276349

7519472791

1618606717

6736731540

966700410:

7109922607

595848:3863

4895813657

1437043510
0.0373107468

0.0377962337

0.0382227062

0.0385894985

0.0388960379

0.0391418457

0.0393265382

0.0394498270

0.0395115195

$n=96$

0.0002192982

0.0013515349

0.0024325786

0.0035104223

0.0045843897

0.0056533772

0.0067162407

0.0077718342

0.0088190167

0.0098566557

0.0108836289

0.0118988250

0.0129011456

0.0138895063

0.0148628375

0.0158200861

0.0167602163

0.0176822109

0.0185850720

0.0194678229

0.0203295083

0.0211691958

0.0219859769

0.0227789676

0.0235473100

0.0242901727

0.0250067517

0.0256962719

0.0263579869

0.0269911808

0.0275951685

0.0281692964

0.0287129433

$0.029225 \% 209$

0.0297064745

0.0301552837

0.0305714629

0.0309545618

0.0313041657

0.0316198965

0.0319014125

0.0321484090

0.0323606189

$0.032: 378124$

0.0326797978

0.0327864215

0.0328 .575682

0.0328931607
3937560243

0193349881

3088360543

4013587066

2715055300

6714956376

6043549120

3165166113

8004770610

4561403509

0565556724

0301058480

7502451778

8477942644

3637145606

8972019234

1191470895

1167706097

8151741141

1988202822

2549847790

7292458482

3390918365

6675032461

8163132671

8464160450

0106401665

7759767582

6277537046

6464755771

8472200913

2711685636

$818802568 j$

8144007028

2916563323

9904768294

0552992516

4255565597

9092463744

9308872911

9454935815

5105561178

0083844395

0115411602

2844846757

4149287708

0688276681

863301804:

8522326545

6196737563

9766492590

257340315i

2110930124

4871187911

7091989724

1381485879

9202407467 
with the machine results was found in all cases to 21 decimals. In addition, the following relation was used as a check on the accuracy of the results

$$
\sum_{k=1}^{n} H_{k}=2 \text {. }
$$

Equation (12) was satisfied to within 2 units in the 21st decimal place for all cases reported here.

In the table only the positive abscissas are reported since all abscissas and weights are symmetric with $x_{-k}=-x_{k}$ and $H_{-k}=H_{k}$.

4. Acknowledgements. This work was sponsored in part by the Air Force Office of Scientific Research of the Office of Aerospace Research, and in part by the United Aircraft Corporation Research Laboratories. The author wishes to thank Miss Anne Putnam for her assistance with the calculations.

Research Laboratories

United Aircraft Corporation

East Hartford 8, Connecticut

1. P. Davis \& P. Rabinowitz, "Abscissas and Weights for Gaussian Quadratures of High Order," NBS J. of Research, v. 56, 1956, p. 35.

2.'P. DAvis \& P. RABINOwitz, "Additional Abscissas and Weights for Gaussian Quadrature of High Order: values for $n=64,80$, and 96," $N B S J$. of Research, v. 60, 1958, p. 613.

3. R. Lobatto, Lessen over de Integraal-Rekening, The Hague, 1852, p. 207.

4. M. R. RADAU, "Etude sur les formules d'approximation qui servant à calculer la valeur numérique d'une intégrale définie." $J$. de Math., (3), v. 6, 1880, p. 283.

5. Ibid, p. 307 .

6. P. Rabinowitz, "Abscissas and Weights for Lobatto Quadrature of High Order," Math. Comp., v. 14, 1960, p. 47.

7. E. T. Whitaker \& G. N. Watson, Modern Analysis, 4th Edition, Cambridge University Press, 1927.

8. H. Szegö, Orthogonal Polynomials, American Math. Soc. Publ., v. 23, American Math. Soc., N. Y., 1939 . 